\title{
Mycobacterium marinum infection with sporotrichoid spread from fish tank exposure
}

\author{
Patrick Fleming MD MSc, Jay S. Keystone MD MSc
}

Cite as: CMAJ 2017 January 16;189:E76. doi: 10.1503/cmaj.151472

A n immunocompetent 52-year-old man presented with lesions and inflammation on his left arm after lacerating his index finger while cleaning a fish tank (Figure 1). The lesions appeared at the laceration site two weeks after the injury and continued to progress for five months. He had no fever or systemic symptoms. On examination, erythematous and ulcerative papulonodules spanned from his left index finger to his upper arm in a sporotrichoid pattern. Laboratory results were normal, including negative bacterial, fungal and mycobacterial tissue cultures (at $30^{\circ} \mathrm{C}$ and $37^{\circ} \mathrm{C}$ ). Skin biopsy showed dermal granulomatous inflammation with multinucleate giant cells, lymphocytes, histiocytes and plasma cells, although direct smears of tissue and special stains for fungi and acid-fast bacilli were negative. Based on the exposure, morphology and histopathology, we diagnosed Mycobacterium marinum infection. Treatment with doxycycline, $100 \mathrm{mg}$ orally twice daily for three months, gave an excellent initial response. Subsequently, the patient was lost to follow-up.

M. marinum is a water-borne pathogen common in both fresh and saltwater fish. ${ }^{1}$ Infection typically occurs on the upper limbs several weeks after injury in aquatic environments or after handling fish. ${ }^{1-3}$ Although lesions are initially solitary or multiple, a linear distribution of papulonodular lesions along lymphatic channels resembling sporotrichosis can occur. The differential diagnosis also includes nocardiosis, leishmaniasis and other mycobacterial or fungal infections. ${ }^{2}$ Results of microbiologic studies are sometimes negative. One case series $(n=29)$ reported positive M. marinum cultures in $41 \%$ of probable infections. ${ }^{3}$ When the results of culture are negative, immunohistochemistry and polymerase chain reaction for mycobacterial 16S rRNA can be helpful. ${ }^{2}$ Systemic symptoms are rare, but deep infections such as septic arthritis and tenosynovitis can occur that require surgical intervention. ${ }^{3}$ Antibiotic treatment alone appears effective in $68 \%-100 \%$ of most noninvasive infections. ${ }^{3}$ It typically includes single- or double-agent coverage with tetracyclines, sulfamethoxazole-trimethoprim, clarithromycin, ethambutol or rifampin for at least three to four months. ${ }^{1-3}$

\section{References}

1. Aubry A, Chosidow O, Caumes E, et al. Sixty-three cases of Mycobacterium marinum infection: clinical features, treatment, and antibiotic susceptibility of causative isolates. Arch Intern Med 2002;162:1746-52.

2. Sia TY, Taimur S, Blau DM, et al. Clinical and pathological evaluation of Mycobacterium marinum group skin infections associated with fish markets in New York City. Clin Infect Dis 2015; Dec. 16. pii: civ937.

3. Johnson MG, Stout JE. Twenty-eight cases of Mycobacterium marinum infection: retrospective case series and literature review. Infection 2015;43:655-62.

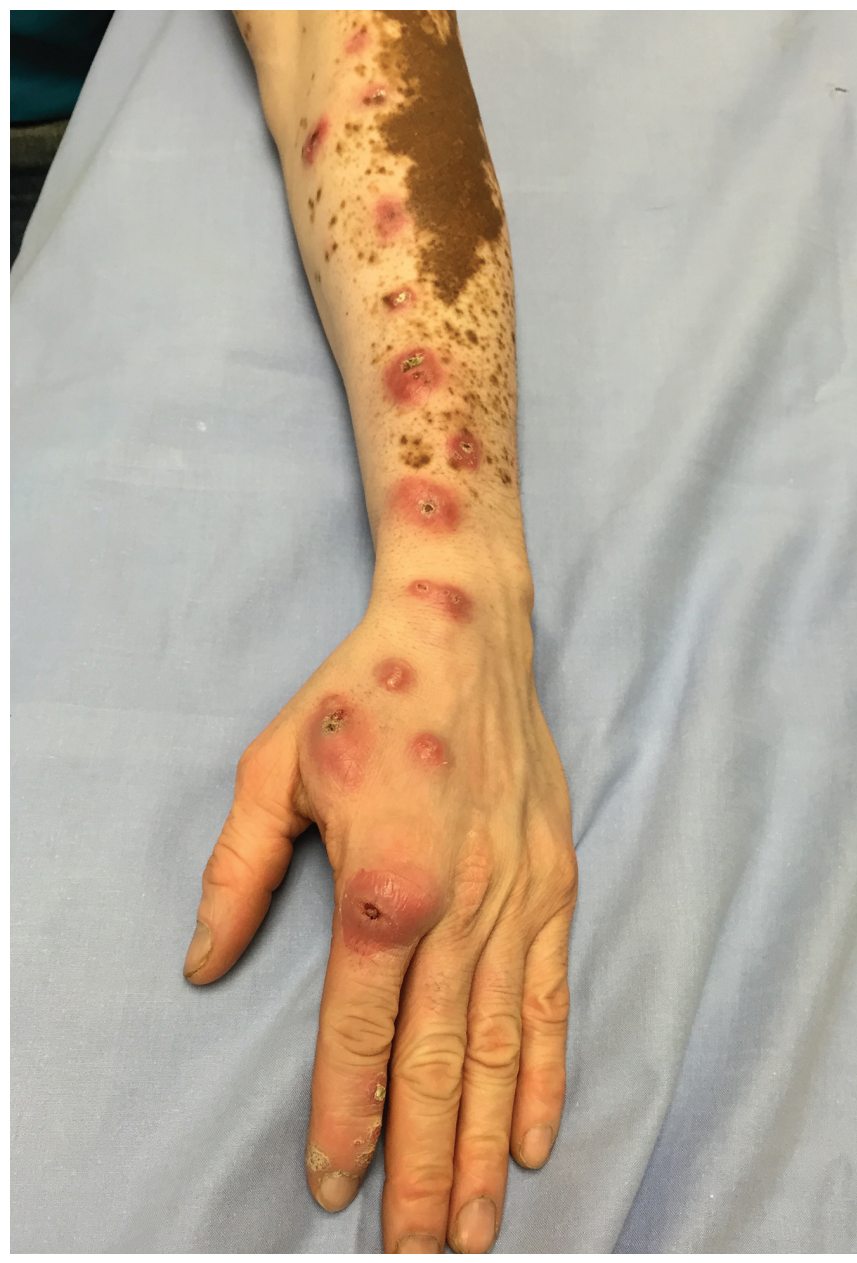

Figure 1: Erythematous and ulcerative papulonodules spanning the left arm in sporotrichoid pattern and diagnosed as Mycobacterium marinum infection in a 52-year-old man after lacerating his left index finger while cleaning a fish tank.

\section{Competing interests: None declared.}

This article has been peer reviewed.

The authors have obtained patient consent.

Affiliations: Division of Dermatology (Fleming), University of Toronto; Tropical Disease Unit, Division of Infectious Diseases (Keystone), Toronto General Hospital, Toronto, Ont.

Correspondence to: Patrick Fleming, flemingp@mun.ca 